\title{
Advection-dominated accretion: A self-similar solution
}

\section{Citation}

Narayan, Ramesh, and Insu Yi. 1994. "Advection-Dominated Accretion: A Self-Similar Solution." The Astrophysical Journal 428 (June): L13. https://doi.org/10.1086/187381.

\section{Permanent link}

http://nrs.harvard.edu/urn-3:HUL.InstRepos:41384942

\section{Terms of Use}

This article was downloaded from Harvard University's DASH repository, and is made available under the terms and conditions applicable to Other Posted Material, as set forth at http:// nrs.harvard.edu/urn-3:HUL.InstRepos:dash.current.terms-of-use\#LAA

\section{Share Your Story}

The Harvard community has made this article openly available.

Please share how this access benefits you. Submit a story. 


\title{
ADVECTION-DOMINATED ACCRETION: A SELF-SIMILAR SOLUTION
}

\author{
Ramesh Narayan and Insu Yi \\ Harvard-Smithsonian Center for Astrophysics, \\ 60 Garden Street, Cambridge, MA 02138
}

\begin{abstract}
We consider viscous rotating accretion flows in which most of the viscously dissipated energy is stored as entropy rather than being radiated. Such advection-dominated flows may occur when the optical depth is either very small or very large. We obtain a family of self-similar solutions where the temperature of the accreting gas is nearly virial and the flow is quasi-spherical. The gas rotates at much less than the Keplerian angular velocity; therefore, the central stars in such flows will cease to spin up long before they reach the break-up limit. Further, the Bernoulli parameter is positive, implying that advection-dominated flows are susceptible to producing outflows. Convection is likely in many of these flows and, if present, will tend to enhance the above effects. We suggest that advection-dominated accretion may provide an explanation for the slow spin rates of accreting stars and the widespread occurrence of outflows and jets in accreting systems.
\end{abstract}

Subject headings: accretion: accretion disks - black hole physics - hydrodynamics

\section{Introduction}

In astrophysical accretion flows, gravitational potential energy is converted to kinetic and thermal energy of the accreting gas. If the thermal energy is efficiently radiated away, the orbiting gas becomes much cooler than the local virial temperature and takes up a thin disk-like configuration. Accretion disk solutions with these characteristics, derived originally by Shakura \& Sunyaev (1973) and Lynden-Bell \& Pringle (1974), have been used to model a variety of accreting systems in astrophysics (see Frank, King, \& Raine 1992 for a review).

We ask here a simple question: What is the nature of the flow if the accreting gas is unable to cool efficiently? We use the term advection-dominated to refer to a flow where the bulk of the liberated thermal energy is carried in by the accreting gas as entropy rather than being radiated. In this Letter we write height-averaged equations to describe advection-dominated flows and discuss the properties of a particular class of self-similar solutions.

\section{Height-Averaged Equations of Advection-Dominated Accretion}

As in the standard theory of thin accretion disks, we vertically average the flow equations and consider a two-dimensional flow in the equatorial $R \phi$ plane. We assume a steady axisymmetric flow so that $\partial / \partial t=\partial / \partial \phi=0$ and all flow variables are functions only of $R$. 
Assuming gas pressure to dominate, we write the pressure as $P=\rho c_{s}^{2}$ where $\rho(R)$ is the height-averaged density and $c_{s}^{2}(R)$ is the isothermal sound speed. We denote the Keplerian angular velocity by $\Omega_{K}(R)=\left(G M / R^{3}\right)^{1 / 2}$ and define the Keplerian velocity $v_{K}=(G M / R)^{1 / 2}$, where $M$ is the central mass. The surface density of the gas is $\Sigma=2 \rho H$, where $H \sim R c_{s} / v_{K}$ is the vertical scale height. Following Shakura \& Sunyaev (1973) we take the kinematic coefficient of shear viscosity to be $\nu=\alpha c_{s} H=\alpha c_{s}^{2} / \Omega_{K}$, where $\alpha$ is a constant. Purely on dimensional grounds, this approximation seems to be particularly appropriate for the self-similar flows discussed here.

The density of the gas $\rho$, its radial velocity $v$, angular velocity $\Omega$, and isothermal sound speed $c_{s}$, satisfy the following four differential equations, namely the continuity equation, the radial and azimuthal components of the momentum equation, and the energy equation (e.g. Abramowicz et al. 1988, Narayan \& Popham 1993):

$$
\begin{gathered}
\frac{d}{d R}(\rho R H v)=0 \\
v \frac{d v}{d R}-\Omega^{2} R=-\Omega_{K}^{2} R-\frac{1}{\rho} \frac{d}{d R}\left(\rho c_{s}^{2}\right) \\
v \frac{d\left(\Omega R^{2}\right)}{d R}=\frac{1}{\rho R H} \frac{d}{d R}\left(\frac{\alpha \rho c_{s}^{2} R^{3} H}{\Omega_{K}} \frac{d \Omega}{d R}\right) \\
\Sigma v T \frac{d s}{d R}=\frac{3+3 \epsilon}{2} 2 \rho H \frac{d c_{s}^{2}}{d R}-2 c_{s}^{2} H v \frac{d \rho}{d R}=Q^{+}-Q^{-} .
\end{gathered}
$$

In equation (4), the left-hand side is the advected entropy, where $T$ is the temperature and $s$ the entropy, while the right-hand side gives the difference between the energy input per unit area due to viscous dissipation $\left(Q^{+}\right)$and the energy loss through radiative cooling $\left(Q^{-}\right)$. For convenience we have defined a parameter $\epsilon=(5 / 3-\gamma) /(\gamma-1)$, where $\gamma$ is the ratio of specific heats; note that $\epsilon=0$ in the limit $\gamma=5 / 3$ and $\epsilon=1$ when $\gamma=4 / 3$. Substituting the viscous dissipation rate for $Q^{+}$, we obtain

$$
Q^{+}-Q^{-}=\frac{2 \alpha \rho c_{s}^{2} R^{2} H}{\Omega_{K}}\left(\frac{d \Omega}{d R}\right)^{2}-Q^{-} \equiv f \frac{2 \alpha \rho c_{s}^{2} R^{2} H}{\Omega_{K}}\left(\frac{d \Omega}{d R}\right)^{2}
$$

The parameter $f$ measures the degree to which the flow is advection-dominated. In the extreme limit of no radiative cooling, we have $f=1$, while in the opposite limit of very efficient cooling, $f=0$. Finally, we define $\epsilon^{\prime} \equiv \epsilon / f$. This is an important parameter which plays a critical role in determining the nature of the flow.

Let us for simplicity assume that $\epsilon^{\prime}$ is independent of $R$. Equations (1)-(4) then permit a self-similar solution of the form

$$
\rho \propto R^{-3 / 2}, \quad v \propto R^{-1 / 2}, \quad \Omega \propto R^{-3 / 2}, \quad c_{s}^{2} \propto R^{-1},
$$

where

$$
v=-\left(5+2 \epsilon^{\prime}\right) \frac{g\left(\alpha, \epsilon^{\prime}\right)}{3 \alpha} v_{K} \approx-\frac{3 \alpha}{\left(5+2 \epsilon^{\prime}\right)} v_{K}
$$




$$
\begin{gathered}
\Omega=\left[\frac{2 \epsilon^{\prime}\left(5+2 \epsilon^{\prime}\right) g\left(\alpha, \epsilon^{\prime}\right)}{9 \alpha^{2}}\right]^{1 / 2} \Omega_{K} \approx\left[\frac{2 \epsilon^{\prime}}{5+2 \epsilon^{\prime}}\right]^{1 / 2} \Omega_{K}, \\
c_{s}^{2}=\frac{2\left(5+2 \epsilon^{\prime}\right)}{9} \frac{g\left(\alpha, \epsilon^{\prime}\right)}{\alpha^{2}} v_{K}^{2} \approx \frac{2}{5+2 \epsilon^{\prime}} v_{K}^{2}, \\
g\left(\alpha, \epsilon^{\prime}\right) \equiv\left[1+\frac{18 \alpha^{2}}{\left(5+2 \epsilon^{\prime}\right)^{2}}\right]^{1 / 2}-1 .
\end{gathered}
$$

The density $\rho$ may be obtained from the mass accretion rate, $\dot{M}=-4 \pi R H v \rho$. In equations (7)-(9), the first relation gives the exact solution, while the second corresponds to the limit when $\alpha^{2} \ll 1$. If we include bulk viscosity through an $\alpha$ prescription there are corrections of order $\alpha^{2}$ in the results. Also, if $\epsilon<0$, i.e. if $\gamma>5 / 3$, there is a second class of self-similar rotating wind solutions where $v>0$. We do not discuss these extensions here.

\section{Properties of the Self-Similar Solution}

In the limit of very efficient cooling, $f \rightarrow 0$ and $\epsilon^{\prime} \rightarrow \infty$, and the solution given in equations (7)-(9) corresponds to a standard thin accretion disk with $v, c_{s} \ll v_{K}$ and $\Omega \rightarrow \Omega_{K}$. The properties of such disks have been widely discussed in the literature. In this Letter we are interested in the opposite limit of advection-dominated flows where $f$ is a reasonable fraction of unity and $\epsilon^{\prime} \sim \epsilon<1$. In this limit, the self-similar solution derived above has several interesting properties which we now discuss. Although our discussion is based on this particular solution, we believe that the results are valid for general advectiondominated accretion flows.

Equation (9) shows that the sound speed in advection-dominated flows is comparable to the Keplerian speed $v_{K}$, which means that the temperature of the accreting gas is nearly virial. This is of course expected since by assumption the gas has no way to cool. When $c_{s} \sim v_{K}$, the disk vertical thickness $H$ is comparable to $R$ and the flow is quasi-spherical. Unfortunately, the vertical averaging on which equations (1)-(4) are based is suspect when $H / R \sim 1$, and therefore some of the numerical factors in our results may be in error.

The radial velocity of the accreting gas is proportional to $\alpha$. This is because the radial speed is determined principally by how fast the viscosity can move angular momentum outwards. Since $v \sim \alpha c_{s}^{2} / v_{K}$, the radial velocity tends to be much larger in advectiondominated flows than in thin disks.

When $\epsilon^{\prime}$ is small, we see that the angular velocity $\Omega$ of the flow is smaller than the local Keplerian $\Omega_{K}$ by a factor $\sim\left(\epsilon^{\prime}\right)^{1 / 2}$. Indeed, as $\epsilon \rightarrow 0$, which corresponds to $\gamma \rightarrow 5 / 3$, $\Omega$ goes to zero and our solution matches on to the Bondi spherical accretion solution for this $\gamma$. (In a sense, the solution (7)-(9) is a natural extension of self-similar $\gamma=5 / 3$ Bondi accretion to rotating flows and general $\gamma$.) Because advection-dominated accretion has a significantly sub-Keplerian $\Omega$, if such a flow were to be present around an accreting star, the star would cease spinning up long before the rotation rate approached the break-up limit. This may have important implications for the spin rate of accreting stars.

Equation (2) shows that the self-similar solution satisfies the relation

$$
\frac{1}{2} v^{2}+\Omega^{2} R^{2}-\Omega_{K}^{2} R^{2}+\frac{5}{2} c_{s}^{2}=0
$$


From this we can compute the normalized parameter $b \equiv B e / v_{K}^{2}$, where $B e$ is the Bernoulli constant:

$$
\begin{aligned}
b & =\frac{1}{v_{K}^{2}}\left(\frac{1}{2} v^{2}+\frac{1}{2} \Omega^{2} R^{2}-\Omega_{K}^{2} R^{2}+\frac{\gamma}{\gamma-1} c_{s}^{2}\right) \\
& =-\frac{\Omega^{2} R^{2}}{2 v_{K}^{2}}+\left(\frac{\gamma}{\gamma-1}-\frac{5}{2}\right) \frac{c_{s}^{2}}{v_{K}^{2}}=\frac{3 \epsilon-\epsilon^{\prime}}{5+2 \epsilon^{\prime}} .
\end{aligned}
$$

As is well-known, $B e$ is conserved in adiabatic flows in the absence of viscosity. Therefore, whenever $b$ is positive, it implies that if we were somehow to turn the gas around and to let it flow adiabatically outward on a radial trajectory, the gas would reach infinity with a net positive energy. On the other hand, if $b<0$, the gas cannot spontaneously escape to infinity. Equation (12) shows that $b$ is positive in advection-dominated flows for all values of $\alpha$ and for any $\gamma<5 / 3$ so long as $f>1 / 3$. Winds, jets and other outflows are common in several accreting systems in astrophysics and it is tempting to speculate that these may originate in advection-dominated flows.

Note that the positivity of the Bernoulli constant does not imply a lack of conservation of energy, but simply arises because viscous stresses transfer energy from small to large radii. Even in the standard theory of thin accretion disks, the energy radiated from any given annulus of the disk is greater by a factor of 3 than the net gravitational energy released within that annulus (e.g. Frank et al. 1992). A similar phenomenon occurs in our solutions, and the excess $b$ at any radius represents energy transferred to that radius from smaller radii. When proper boundary conditions are applied and the full global problem is solved, total energy will of course be conserved.

It is easily shown that the entropy increases inwards in our self-similar solution. We need therefore to consider the possibility of convective energy transport. In a rotating medium, the condition for a dynamical convective instability is

$$
N_{e f f}^{2} \equiv N^{2}+\kappa^{2}=-\frac{1}{\rho} \frac{d P}{d R} \frac{d \ln s}{d R}+\kappa^{2}<0
$$

where $N$ is the usual Brunt-Väisälä frequency and $\kappa$ is the epicyclic frequency which in our case is equal to $\Omega$. For our self-similar solution we find (in the limit when $\alpha^{2} \ll 1$ )

$$
N_{\text {eff }}^{2}=\frac{10 \epsilon^{\prime}+6 \epsilon \epsilon^{\prime}-15 \epsilon}{(5+3 \epsilon)\left(5+2 \epsilon^{\prime}\right)} \Omega_{K}^{2} .
$$

This gives a dynamical instability for

$$
f>\frac{2}{3}+\frac{2}{5} \epsilon
$$

Even when $f$ is below this limit a mild double diffusive instability will be present.

Although advection-dominated flows are convectively unstable, we can show that convection, if at all, only strengthens our main conclusions. The height-integrated convective energy flux $F_{c}$ in the presence of an unstable entropy gradient can be written quite generally as

$$
F_{c}=-\Sigma K_{c} T \frac{d s}{d R}
$$


where $K_{c}$ is the diffusion constant associated with convective transport. In the spirit of the Shakura-Sunyaev viscosity formula let us write $K_{c}=\alpha_{c} c_{s}^{2} / \Omega_{K}$. Substituting our selfsimilar solution into equation (16) we then find that $F_{c}$ scales as $R^{-2}$ and has a negative divergence. Convection therefore provides an additional energy source in equation (4) of magnitude

$$
Q_{c}^{+}=-\vec{\nabla} \cdot \vec{F}=-\Sigma \frac{\alpha_{c} c_{s}^{2}}{v_{K}} T \frac{d s}{d R}
$$

This term has the same structure as the left-hand side of equation (4). We can therefore combine the two terms by simply redefining $\epsilon^{\prime}$ to be

$$
\epsilon^{\prime}=\frac{\epsilon}{f}\left(1+\frac{\alpha_{c} c_{s}^{2}}{v v_{K}}\right)=\frac{\epsilon}{f}\left(1-\frac{2}{3} \frac{\alpha_{c}}{\alpha}\right) .
$$

With this definition, all of the results in equations (6)-(10) continue to hold.

In general we expect $\alpha \gtrsim \alpha_{c}$. This is because convective turbulence contributes to the viscosity but not all sources of viscosity produce bulk energy transport. (Ryu \& Goodman (1992) show that if the entropy in an accretion disk is stratified vertically, then convection actually moves angular momentum inwards; in our case we have a radially stratified medium and so we expect convection to produce a normal outwardly-oriented angular momentum flux.) Since $\alpha \gtrsim \alpha_{c}$, equation (18) shows that convection only introduces a modest perturbation to the energy equation (4). This is very different from the situation in stellar interiors where convection, once it sets in, strongly modifies the structure and essentially forces an isentropic radial distribution. In our case, the strong advection ensures that convection can only be a moderate perturbation. Indeed, as equation (18) shows, the primary effect of convection is to reduce the value of the parameter $\epsilon^{\prime}$. This means that the various properties of advection-dominated flows discussed earlier become even more pronounced. In particular, when there is convection, the parameter $b$ remains positive for a wider range of $f$ than in the non-convective case. The reason is that convection transports energy from smaller to larger $R$ and therefore adds to the effect of the viscous stress.

The above discussion suggests that the main results of this paper are likely to survive even if there are other instabilities in the flow.

\section{Discussion}

Advection-dominated accretion is expected to occur in several astrophysical situations. Thin accretion disks for example exhibit a thermal instability at sufficiently low optical depth, when the cooling through free-free emission is unable to keep up with the viscous energy generation. Numerical models of accretion disks in cataclysmic variables (Narayan \& Popham 1993) show that, under the influence of this instability, the disk switches to an advection-dominated flow where only a fraction of the released energy is radiated. A more extreme possibility is that at very low accretion rates the infalling material may never cool sufficiently to collapse to a thin disk, and we could imagine an advection-dominated flow all the way from the outermost radius down to the central star or black hole.

Advection can dominate also in the opposite limit when the mass accretion rate is very high and the optical depth $\tau$ of the disk is large. If the cooling time of the disk, $t_{\text {cool }} \sim H \tau / c$, is longer than the accretion time $R / v$, most of the accretion energy is retained in the gas and we have an advection-dominated flow. Such conditions are possible 
in young stellar objects and symbiotic stars in outburst (Popham et al. 1993) and in accreting neutron stars and black holes at high mass accretion rates.

Thus, advection-dominated accretion is likely to occur in a number of astrophysical systems, and some objects may have both advection-dominated and standard coolingdominated zones at different radii. Surprisingly, very few studies in the literature have included advection effects, and the physics of advection-dominated accretion has been hardly discussed at all. The self-similar solutions we present in $\S 2$ of this Letter represent a first step towards understanding such flows.

Our self-similar solutions have some interesting features which we have described in

$\S 3$. Here we would like to highlight the following:

1. The angular velocity $\Omega$ of the flow is less than the Keplerian angular velocity $\Omega_{K}$; in fact, for the astrophysically interesting case of $\gamma \rightarrow 5 / 3(\epsilon \rightarrow 0)$, we find $\Omega \ll \Omega_{K}$. In accretion theory it is usually assumed that the infalling material rotates with $\Omega \sim \Omega_{K}$ and that accreting stars will spin up to the "break-up limit" corresponding to a stellar spin rate $\Omega_{*} \sim \Omega_{K}\left(R_{*}\right)$, where $R_{*}$ is the equatorial radius of the star. Advectiondominated accretion flows are very different and the star may reach an equilibrium spin state with $\Omega_{*} \ll \Omega_{K}\left(R_{*}\right)$. At the equilibrium $\Omega_{*}$ the system will adjust the angular momentum flux in such a way that $\Omega_{*}$ remains constant (Popham \& Narayan 1991, Paczyński 1991). Similarly, in the case of accretion onto a magnetic star it is usually assumed that the central star spins up until $\Omega_{*}=\Omega_{K}\left(R_{A}\right)$, where $R_{A}$ is the Alfven radius. Once again, if the accretion is advection-dominated, we can have $\Omega_{*} \ll \Omega_{K}\left(R_{A}\right)$.

2. The scaled Bernoulli parameter $b$ (see equation 12) is positive in self-similar advectiondominated flows for a wide range of parameters. We have argued that this means the gas in such flows is capable of spontaneously escaping to infinity. We suggest that advection-dominated flows may provide a generic explanation for many of the outflows and jets that are so ubiquitous in accreting systems. It is beyond the scope of this Letter to discuss the exact mechanism by which outflows may be generated. One possibility is that a shock may divert part of the high entropy accreting material into an outward-pointing trajectory. Alternatively, the positivity of $b$ may make the generation of winds so easy that even a quite modest radiative or magnetic stress may set off a substantial wind. (We must caution however that, in some circumstances, once the material reaches the surface of the disk it may cool and lose its positive $b$, perhaps making it harder to generate a wind.)

3. By definition, the radiative luminosity of an accretion-dominated flow is much less than the standard $G M \dot{M} / R_{*}$ that is usually associated with accretion. Therefore, estimates of the mass accretion rates of observed systems could be seriously in error, especially when the central star is a black hole which can swallow the advected entropy, or when much of the accretion energy escapes as an outflow. Moreover, since the accreting gas is almost at virial temperature, the spectrum is likely to be much hotter than the spectrum of an equivalent thin disk. This is particularly true of optically thin advection-dominated flows.

4. Advection-dominated flows are convectively unstable. Because convection transfers energy from small to large radii, we find that it enhances the effects described above. The self-similar solutions described here technically extend from $R=0$ to $R=\infty$ with no boundaries. How relevant are the results if the accretion flow is advection-dominated only over a finite range of $R$ and is bounded on the outside and inside by non-advection zones? We have carried out some preliminary investigations of this question by numerically solving equations (1)-(4) for various boundary conditions. Figure 1 shows an example 
where the accreting gas starts off as a thin disk at large $R$, goes through an advectiondominated phase, and becomes a rotating settling "star" at small $R$. Over a range of intermediate radii the numerical solution is close to the self-similar form. We have calculated other numerical solutions where the central star is a black hole and the flow goes through a sonic point, and again we find that the flow is nearly self-similar in between the two boundaries.

From these numerical examples we conclude that the self-similar solutions we have described may be of more than academic interest and that perhaps real flows often resemble the self-similar form. In any case, the main features of these solutions, namely slow rotation and the ability to make outflows, are probably generic to a wide class of advection-dominated flows.

Acknowledgements: RN thanks Bob Popham for numerous discussions on the physics of accretion, advection and spin-up. We are grateful to Lee Hartmann for helpful comments and the referee for useful criticism. This work was supported in part by NSF grant AST 9148279 .

\section{References}

Abramowicz, M., Czerny, B., Lasota, J. P., \& Szuszkiewicz, E. 1988, ApJ, 332, 646

Frank, J., King, A., \& Raine, D. 1992, Accretion Power in Astrophysics (Cambridge, UK: Cambridge University Press)

Lynden-Bell, D., \& Pringle, J. E. 1974, MNRAS, 168, 603

Narayan, R., \& Popham, R. 1993, Nature, 362, 820

Paczyński, B. 1991, ApJ, 370, 597

Popham, R., \& Narayan, R. 1991, ApJ, 370, 604

Popham, R., Narayan, R., Hartmann, L., \& Kenyon, S. 1993, ApJ, 415, L127

Ryu, D., \& Goodman, J. 1992, ApJ, 388, 438

Shakura, N. I., \& Sunyaev, R. A. 1973, A\&A, 24, 337

\section{Figure Caption}

Figure 1. A numerical solution of equations (1)-(4) with $G M=1, \alpha=0.1, f=1$, and $\epsilon=1 / 3$ (or $\gamma=1.5$ ). At $R=10^{6}$ (the outer edge), the boundary conditions are $\Omega=\Omega_{K}$ and $c_{s}=0.001 v_{K}$, appropriate to a very thin disk. At $R=1$ (the inner edge), the boundary condition is $v=0.05 c_{s}$, corresponding to a settling star. The "star" in this example extends out to about $\log (R) \sim 1$. The angular momentum flux has been set to zero, corresponding approximately to an equilibrium spinup state. (The precise equilibrium value of this parameter depends on the structure of the central star, cf Popham \& Narayan 1991). The solid lines in the three panels show the radial velocity $v$, the scaled angular velocity $\Omega / \Omega_{K}$, and the scaled Bernoulli parameter $b$. The dashed lines show the results corresponding to the self-similar solution. 
This figure "fig1-1.png" is available in "png" format from: http://arXiv.org/ps/astro-ph/9403052v1 